\title{
An action principle for the quantization of parametric theories and nonlinear quantum cosmology
}

\author{
Charles H-T Wang, Smaragda Kessari and Edward R Irvine \\ Department of Physics, Lancaster University \\ Lancaster LA1 4YB, UK \\ E-mail: c.wang@lancaster.ac.uk, \\ s.kessari@lancaster.ac.uk, e.irvine@lancaster.ac.uk
}

\begin{abstract}
By parametrizing the action integral for the standard Schrödinger equation we present a derivation of the recently proposed method for quantizing a parametrized theory. The reformulation suggests a natural extension from conventional to nonlinear quantum mechanics. This generalization enables a unitary description of the quantum evolution for a broad class of constrained Hamiltonian systems with a nonlinear kinematic structure. In particular, the new theory is applicable to the quantization of cosmological models where a chosen gravitational degree of freedom acts as geometric time. This is demonstrated explicitly using three cosmological models: the Friedmann universe with a massless scalar field and Bianchi type I and IX models. Based on these investigations, the prospect of further developing the proposed quantization scheme in the context of quantum gravity is discussed.
\end{abstract}

PACS numbers: 04.60.Ds, 04.20.Fy, 04.60.Kz, 11.10.Lm

\section{Introduction}

The Hamiltonian formalism and canonical quantization of Einstein's theory of gravitation was originally developed by Dirac [1, Arnowitt, Deser, Misner (ADM) [2, Wheeler [3], DeWitt 4] and others. The Dirac-ADM action for general relativity is constructed by representing the spacetime metric using the spatial metric components together with the lapse and shift functions that specify a time foliation. The latter set of functions enter into the action principle as Lagrangian multipliers ensuring the general covariance of the theory. Indeed, the 'already parametrized' form of the Dirac-ADM action requires that not all three-metric components and their conjugate momenta are to be freely specified on a spatial Cauchy surface. The Hamiltonian and momentum constraints must be satisfied initially and will be dynamically preserved [5. A major challenge in quantizing general relativity is the treatment of these constraints. The straightforward application of the Dirac constraint quantization leads to a functional form of the Klein-Gordon equation, the Wheeler-DeWitt equation, and the associated difficulties in admitting a probabilistic interpretation. An alternative approach, the ADM 
quantization method, attempts to circumvent these problems by isolating four out of the six spatial metric components (and their momenta) and regarding them as the evolution and coordinatization parameters, known as the embedding variables [6, 8, 9, for the quantum evolution of the remaining 'true' gravitational degrees of freedom. However different set of embedding variables can result in inequivalent quantizations. A further drawback of this approach is that the reduced Hamiltonian operator may involve a square root of an indefinite expression and therefore may not be Hermitian.

The above pioneering work was followed by Kuchař's significant contributions to canonical quantum gravity in which Dirac quantization and the use of embedding variables are assimilated [7, 8, 9]. Guided by the analogy with parametrized field theory, a canonical transformation is sought such that in the new set of variables the Hamiltonian and momentum constraints are linear in the conjugate momenta of the embedding variables [10, 11]. Remarkably, if this 'Kuchar transformation' exists then Dirac and ADM quantization in fact coincide, with both methods leading to the same functional Schrödinger equation for the quantum evolution of the non-embedding variables. Furthermore, this approach holds out the possibility of constructing a framework for quantum geometrodynamics with the genuine Lie algebra of spacetime diffeomorphism. This was meticulously investigated by Isham and Kuchař [12]. A stringent criterion for Kuchař's embedding variables is that they must be spacetime scalars, to guarantee the integrability of quantum evolution and its independence of time slicing [1]. Nevertheless, Kuchař successfully demonstrated that in certain midisuperspace models, i.e. simple field-theoretical models with Killing symmetries, it is possible to find embedding variables explicitly that fulfill this requirement 17, 13. Unfortunately, for some other important models, notably the 'scalar geons' 14, 15, 16, 17, finding the embedding variables has proven to be a formidable task.

Recently, Hájiček and Kijowski developed a new approach in which the local existence of covariant gauge fixings is exploited to effect the decomposition of canonical variables on the constraint surface (defined by the Hamiltonian and momentum constraints) into embedding and dynamical variables [19, 20. The resulting 'Kuchař decomposition' may form a basis for a complete Kuchař transformation for a large class of geometries. The usefulness of this approach has been demonstrated by Hájiček and Kiefer in studying the quantum dynamics of a collapsing shell of null dust with spherical symmetry [21] derived from the 'Louko-Whiting-Friedman model' 22. However if a full theory of quantum gravity is to be constructed based on the covariant gauge fixing description then some obstructions will be encountered as have been noted in [20]: A complete Kuchař transformation becomes unattainable for geometries with Killing symmetries, which has also been observed by Torre [23]. Besides, different gauges can lead to inequivalent quantum theories 24] and global gauge fixing may not exist at all [18, 25].

The purpose of this paper is to present an alternative approach to the quantization of a parametric theory, with a view to quantizing gravity, that will complement the above developments. While much insight has been gained from the quantization of a theory parametrized at the classical level, guidance for quantum gravity gleaned this way appears to be limited. Apart from technical difficulties and topological restrictions of the explicit constructions of Kuchař's embedding variables, there is a conceptual issue concerning these preferred variables. This is part of what has been attributed to the 'problem(s) of time' [11, 26]. More specifically, the existence of a functional time relies on the canonical transformation for the geometrodynamical variables: $\left(g_{a b}, p^{a b}\right) \rightarrow\left(X^{A}, P_{A}, q^{r}, p_{r}\right)$ that splits the Hamiltonian and momentum constraints $\mathcal{H}_{A}\left(X^{B}, P_{B}, q^{r}, p_{r}\right)$ into a 'kinematic part' $\mathcal{H}_{A}^{\mathrm{K}}\left(X^{B}, P_{B}\right)$ and a 'dynamic part' $\mathcal{H}_{A}^{\mathrm{D}}\left(X^{B}, q^{r}, p_{r}\right)$, i.e.

$$
\mathcal{H}_{A}\left(X^{B}, P_{B}, q^{r}, p_{r}\right)=\mathcal{H}_{A}^{\mathrm{K}}\left(X^{B}, P_{B}\right)+\mathcal{H}_{A}^{\mathrm{D}}\left(X^{B}, q^{r}, p_{r}\right) .
$$

(Here $a, b=1,2,3 ; A, B=0,1,2,3 ; \quad r=1,2$.) A crucial assumption made by Kuchař is that $\mathcal{H}_{A}^{\mathrm{K}}\left(X^{B}, P_{B}\right)$ are linear in $P_{A}$ so that the theory is 'deparametrizable' with $X^{A}$ and $q^{r}$ identified 
as embedding and dynamical variables respectively. However an obstacle typical of midisuperspace models that prevents deparametrization is the nonlinear nature of $\mathcal{H}_{A}^{\mathrm{K}}\left(X^{B}, P_{B}\right)$. Although in some cases, an ingenious choice of $X^{A}$ may well be the way out, this can be technically infeasible and might not be possible in principle. In these circumstances, we adopt the viewpoint that it may be possible to formulate a parametric quantum evolution of the dynamical variables $q^{r}$ and treat the remaining variables $X^{A}$ as unquantized kinematic variables without further restrictions. The new formulation sought should, nevertheless, reduce to Dirac quantization and is expected to be equivalent to certain other schemes up to a factor ordering [27] (in a semiclassical limit) if Kuchař transformation exists that reduces $\mathcal{H}_{B}^{\mathrm{K}}\left(X^{A}, P_{A}\right)$ to simply $P_{A}$.

As a first step towards the fully quantum field-theoretical formulation of our approach, a quantum mechanical description is adopted. In this simplified picture it is possible to focus on conceptual issues while establishing the essential methodology with a view to further generalization. Following a recent publication [28] where a new quantization scheme was proposed, we report in this paper how the proposed scheme can arise from a direct parametric description of quantum evolution as opposed to seeking to quantize a parametrized classical theory in a conventional manner. In section 2. a parametric form of quantum evolution equations is introduced based on a parametrized action principle for the Schrödinger equation describing a non-relativistic particle. The advantage of this reformulation is that it opens up new avenues for generalized parametric quantum evolution beyond the description of a Newtonian particle. The generalized method is applied in section 3 to a simple Friedmann universe filled with a massless scalar field. Two further cosmological models, of Bianchi types I and IX, both involving gravity only are analyzed in sections 4 using the nonlinear quantization scheme. Conclusions and discussions are made in section 5 where speculations on future work are discussed. Units in which $c=\hbar=16 \pi G=1$ are adopted throughout.

\section{Parametrized quantum evolution of a non-relativistic particle}

Consider a non-relativistic quantum particle of unit mass moving in an $n$-dimensional Riemannian manifold $M$ with a time-dependent metric and coordinates $q^{a},(1 \leq a \leq n)$ subject to a potential $V\left(q^{a}, T\right)$. In terms of the Newtonian time $T$, the weight $\frac{1}{2}$ wavefunction $\Psi\left(q^{a}, T\right)$ of the particle satisfies the Schrödinger equation

$$
\mathrm{i} \frac{\partial \Psi}{\partial T}=\hat{h} \Psi
$$

where the $\hat{h}=\hat{h}(T)$ denotes the time-dependent Hamiltonian operator

$$
\hat{h}(T)=-\frac{1}{2} \Delta+V
$$

with $\Delta$ denoting the Laplace-Beltrami operator on weight $\frac{1}{2}$ functions on $M$ [10, 28].

It has long been known that, in terms of the equal time inner product

$$
\left\langle\Psi_{1}, \Psi_{2}\right\rangle:=\int \Psi_{1}^{*} \Psi_{2} \mathrm{~d}^{n} q
$$

of wavefunctions $\Psi_{1}$ and $\Psi_{2}$, the Schrödinger equation (2) can be derived from the action integral (e.g. [29]):

$$
S_{\mathrm{Q}}\left[\Psi, \Psi^{*}\right]=\int \mathrm{d} T \Re\left\langle\Psi,\left(\mathrm{i} \partial_{T}-\hat{h}\right) \Psi\right\rangle
$$


by freely varying $\Psi$ and its complex conjugate $\Psi^{*} .{ }^{1}$ However the potential significance of this formulation in the context of constrained Hamiltonian systems does not appear to have been fully recognized in the literature and will be explored in this paper. In what follows an action integral equivalent to (5) will be constructed that generates quantum evolution equations with respect to a general time coordinate, by parametrizing the Newtonian time and turning it into a constrained classical variable.

We anticipate the parametrized $T$ and its momentum to couple with a reformulated Schrödinger equation in a manner similar to the interaction between classical and quantum variables. Of course, the 'classical variables' arising from this procedure do not carry additional physical degrees of freedom due to the constraining relations accompanying the parametrized theory. Therefore these 'constrained classical variables' are really kinematic (embedding) variables, rather than genuine dynamical variables, forging a link between the physical and geometrical descriptions of the systems [28. Nonetheless the expected mathematical similarity with the semiclassical theory suggests that the action integral of the parametrized quantum theory is a sum of its kinematic and dynamic parts. While the kinematic part depends on 'classical variables' only the dynamic part will involve both classical and quantum variables derived from the original action (5), whose solutions have the scaling invariance under $\Psi \rightarrow \lambda \Psi$ for any complex constant $\lambda$, even though $|\lambda| \neq 1[30$. However this scaling invariance will apparently be violated due to the presence of the kinematic part of the action that is independent of the quantum variables. A way to avoid this problem is to eliminate the scaling invariance by using, instead of (5), the modified action integral [30, 31]:

$$
S_{\mathrm{Q}}\left[\Psi, \Psi^{*}, \alpha\right]=\int \mathrm{d} T\left\{\Re\left\langle\Psi, \mathrm{i} \partial_{T} \Psi\right\rangle-\langle\Psi, \hat{h} \Psi\rangle+\alpha(\langle\Psi, \Psi\rangle-1)\right\}
$$

with $\alpha=\alpha(T)$. With respect to this Lagrangian multiplier, the variation of (6) enforces the normalization condition

$$
\langle\Psi, \Psi\rangle=1
$$

for the wave function $\Psi$, whereas the variation with respect to $\Psi$ and $\Psi^{*}$ yields the Schrödinger equation of the form

$$
\mathrm{i} \frac{\partial \Psi}{\partial T}=(\hat{h}-\alpha) \Psi
$$

Despite that $\alpha$ can be specified arbitrarily, different choices of it correspond to wavefunctions unitarily related by a phase depending only on time and therefore considered as physically equivalent.

The action (6) will now be brought to a parametric form. This procedure starts by expressing $T$ as an arbitrarily chosen function of a parameter time $t$ to yield

$$
S_{\mathrm{Q}}\left[\Psi, \Psi^{*}, \alpha\right]=\int \mathrm{d} t\left\{\Re\left\langle\Psi, \mathrm{i} \partial_{t} \Psi\right\rangle-\dot{T}[\langle\Psi, \hat{h} \Psi\rangle-\alpha(\langle\Psi, \Psi\rangle-1)]\right\}
$$

where the over dot denotes $\partial_{t}$. Note that in this expression $\Psi=\Psi\left(q^{a}, t\right):=\Psi\left(q^{a}, T(t)\right), \alpha=\alpha(t):=$ $\alpha(T(t))$ and $\hat{h}=\hat{h}(t):=\hat{h}(T(t))$ (with slight abuse of notation). At this stage $T(t)$ is merely an externally supplied function. It can, however, be included as part of an enlarged set of dependent variables augmented with a new pair of Lagrangian multiplier and constraint. To this end introduce the variable $\Pi(t)$ and use it to define the quantity

$$
\mathcal{H}_{\mathrm{Q}}:=\Pi+\langle\Psi, \hat{h} \Psi\rangle-\alpha(\langle\Psi, \Psi\rangle-1)
$$

\footnotetext{
${ }^{1}$ The subscript 'Q' indicates 'quantum'.
} 
so that, if $\mathcal{H}_{\mathrm{Q}}$ vanishes, $\Pi$ equals the coefficient of $\dot{T}$ in the action thereby serving as the momentum of $T(t)$. By replacing the coefficient of $\dot{T}$ with $\Pi$ in the Lagrangian for (9) and adjoining this Lagrangian with the term $-N \mathcal{H}_{\mathrm{Q}}$ using a Lagrangian multiplier $N=N(t)$, we are led to the following action

$$
S_{\mathrm{Q}}\left[\Psi, \Psi^{*}, \alpha, T, \Pi, N\right]=\int \mathrm{d} t\left\{\Re\left\langle\Psi, \mathrm{i} \partial_{t} \Psi\right\rangle+\Pi \dot{T}-N \mathcal{H}_{\mathrm{Q}}\right\}
$$

This action takes a parametric form and is equivalent to (9) upon variation with respect to all components in the extended set of variables $\left\{\Psi, \Psi^{*}, \alpha, T, \Pi, N\right\}$. It is not hard to further express this action in a complete canonical form by defining the conjugate momenta of $\Psi$ and $\Psi^{*}$. This form is however not required in our present analysis.

It is instructive to split $\mathcal{H}_{\mathrm{Q}}$ into its 'kinematic' part

$$
\mathcal{H}_{\mathrm{Q}}^{\mathrm{K}}=\Pi
$$

and 'dynamic' part

$$
\mathcal{H}_{\mathrm{Q}}^{\mathrm{D}}=\langle\Psi, \hat{h} \Psi\rangle-\alpha(\langle\Psi, \Psi\rangle-1)
$$

so that

$$
\mathcal{H}_{\mathrm{Q}}=\mathcal{H}_{\mathrm{Q}}^{\mathrm{K}}+\mathcal{H}_{\mathrm{Q}}^{\mathrm{D}}
$$

Accordingly, the structure of parametrized action (11) can be made more transparent with a similar split:

$$
S_{\mathrm{Q}}=S_{\mathrm{Q}}^{\mathrm{K}}+S_{\mathrm{Q}}^{\mathrm{D}}
$$

in terms of the kinematic part

$$
S_{\mathrm{Q}}^{\mathrm{K}}[T, \Pi, N]=\int \mathrm{d} t\left\{\Pi \dot{T}-N \mathcal{H}_{\mathrm{Q}}^{\mathrm{K}}\right\}
$$

and the dynamic part

$$
S_{\mathrm{Q}}^{\mathrm{D}}\left[\Psi, \Psi^{*}, \alpha, T, \Pi, N\right]=\int \mathrm{d} t\left\{\Re\left\langle\Psi, \mathrm{i} \partial_{t} \Psi\right\rangle-N \mathcal{H}_{\mathrm{Q}}^{\mathrm{D}}\right\}
$$

The parametrized quantum evolution equations can be explicitly derived from the action (11) under variations with respect to $\Psi$ and its conjugate, $\alpha, T, \Pi$ and $N$ to be

$$
\begin{gathered}
\mathrm{i} \partial_{t} \Psi=N(\hat{h}-\alpha) \Psi \\
\langle\Psi, \Psi\rangle=1 \\
\dot{T}=N \frac{\partial \mathcal{H}_{\mathrm{Q}}}{\partial \Pi} \\
\dot{\Pi}=-N \frac{\partial \mathcal{H}_{\mathrm{Q}}}{\partial T} \\
\mathcal{H}_{\mathrm{Q}}=0
\end{gathered}
$$

respectively. The structure of these equations indeed resembles that of a semiclassical theory. It is evident that the kinematic action $S_{\mathrm{Q}}^{\mathrm{K}}$ involves only 'classical' variables and takes a canonical form. 
This makes it possible to perform a canonical transformation, say, from $(T, \Pi)$ to $\left(T^{\prime}, \Pi^{\prime}\right)$ by preserving the form of $S_{\mathrm{Q}}^{\mathrm{K}}[T, \Pi, N]=S_{\mathrm{Q}}^{\mathrm{K}}\left[T^{\prime}, \Pi^{\prime}, N\right]$. It follows immediately that with these transformed variables, the action $S_{\mathrm{Q}}\left[\Psi, \Psi^{*}, \alpha, T^{\prime}, \Pi^{\prime}, N\right]$ will yield equivalent quantum evolution equations of the same form as (18)-(22) with the substitutions $T \rightarrow T^{\prime}$ and $\Pi \rightarrow \Pi^{\prime}$. This suggests the set of parametric quantum evolution equations is somehow more flexible in choosing a 'time' variable than that of the Schrödinger equation (2) that assumes the existence of a preferred choice of time. A further advantage of the present approach is the implication that the action (11) for a wider class of the kinematic actions with a general $\mathcal{H}_{\mathrm{Q}}^{\mathrm{K}}=\mathcal{H}_{\mathrm{Q}}^{\mathrm{K}}(T, \Pi)$ in place of (12) may play a fundamental role in the quantization of a parametric theory where no preferred (Newtonian) time can be identified. In this case the parametric quantum evolution system (18)-(22) becomes nonlinear and cannot be reduced to the Schrödinger equation (2). The following sections will provide illustrative examples for this generalization based on minisuperspace models.

\section{Friedmann universe with a quantized massless scalar field}

The theoretical framework for the quantization scheme developed in the previous section will now be employed in the quantization of a simple Friedmann universe filled with a massless scalar field. The nonlinear quantization of a similar model with a massive scalar field was addressed in [28] in terms of a nonlinear integro-partial differential system derived from (18)-(22) (for $\alpha=0$ ), which was reduced to a system of nonlinear ordinary differential equations of infinite dimensions. The latter was further truncated for numerical simulation. Not surprisingly, the reduced system can be shown to be non-dissipative since there exists an underlying variational principle as developed in the previous section.

The present Friedmann model with a massless scalar field can be investigated analytically. The classical Lagrangian for this model may be found from [32] and 28] by dropping the mass term to obtain

$$
L(\phi, \dot{\phi}, R, \dot{R}, N)=-\frac{6 R}{N} \dot{R}^{2}+\frac{6 R^{3}}{N} \dot{\phi}^{2}+6 N K R .
$$

Here $\phi=\phi(t)$ is the scalar field $\phi$ which has been conveniently re-scaled. The positive variables $R=$ $R(t)$ and $N=N(t)$ are the scale factor and lapse function respectively appearing in the RobertsonWalker metric

$$
\mathrm{d} s^{2}=-N^{2} \mathrm{~d} t^{2}+R^{2} \mathrm{~d} \ell^{2}
$$

with $\mathrm{d} \ell^{2}$ denoting the squared line element on the homogeneous and isotropic 3 -space, which may be closed $(K=1)$, flat $(K=0)$ or 'open' $(K=-1)$. The momenta conjugate to $R$ and $\phi$ are

$$
\begin{aligned}
\Pi & :=\frac{\partial L}{\partial \dot{R}}=-\frac{12 R}{N} \dot{R} \\
p & :=\frac{\partial L}{\partial \dot{\phi}}=\frac{12 R^{3}}{N} \dot{\phi}
\end{aligned}
$$

It follows that the corresponding Hamiltonian $H(\phi, p, R, \Pi, N):=p \dot{\phi}+\Pi \dot{R}-L$ takes the form $H=N \mathcal{H}$ where

$$
\mathcal{H}(\phi, p, R, \Pi)=-\frac{\Pi^{2}}{24 R}+\frac{p^{2}}{24 R^{3}}-6 K R
$$


The canonical equations of classical motion can be derived with arbitrary $N(t)$. The gauge condition $N=12 R^{3}$ is chosen so that these equations take a simpler form as follows

$$
\begin{gathered}
\dot{\phi}=p \\
\dot{p}=0 \\
\dot{R}=-R^{2} \Pi \\
\dot{\Pi}=-\frac{R \Pi^{2}}{2}+\frac{3 p^{2}}{2 R}+72 K R^{3}
\end{gathered}
$$

subject to the (Hamiltonian) constraint

$$
-\frac{\Pi^{2}}{24 R}+\frac{p^{2}}{24 R^{3}}-6 K R=0
$$

Clearly, from (29) the momentum $p$ is a constant of motion, with which (28) is readily integrated. With a suitable choice of the origin of the coordinate time $t$, one obtains

$$
R^{2}=\frac{|p| e^{2|p| t}}{6\left(1+K e^{4|p| t}\right)}
$$

for $-\infty<t<\infty$ with $K=0,1$ and $-\infty<t<0$ with $K=-1$. In terms of this expression (30) can be easily solved for $\Pi$.

The quantization of this model requires a degree of freedom to be quantized. We choose this to be the scalar field $\phi$, thereby regarding the scale factor as the 'geometric time'. As per discussions in section 2 we split $\mathcal{H}$ by

$$
\mathcal{H}=\mathcal{H}^{\mathrm{K}}+\mathcal{H}^{\mathrm{D}}
$$

where

$$
\begin{gathered}
\mathcal{H}^{\mathrm{K}}=-\frac{\Pi^{2}}{24 R} \\
\mathcal{H}^{\mathrm{D}}=\frac{p^{2}}{24 R^{3}}-6 K R .
\end{gathered}
$$

To proceed the operator

$$
\hat{h}=-\frac{1}{24 R^{3}} \frac{\partial^{2}}{\partial \phi^{2}}-6 K R
$$

is constructed by substituting $p \rightarrow \hat{p}:=-\mathrm{i} \frac{\partial}{\partial \phi}$ into $\mathcal{H}^{\mathrm{D}}$. This operator is Hermitian with respect to the inner product

$$
\left\langle\Psi_{1}, \Psi_{2}\right\rangle:=\int_{-\infty}^{\infty} \Psi_{1}^{*} \Psi_{2} \mathrm{~d} \phi
$$

of any two wavefunctions $\Psi_{1}(\phi, t)$ and $\Psi_{2}(\phi, t)$.

In the gauge $N=12 R^{3}$, the following nonlinear evolution equations for the normalized wavefunction $\Psi(\phi, t)$ and kinematic variables $R(t), \Pi(t)$ then arise from (18)-(22):

$$
\mathrm{i} \frac{\partial \Psi}{\partial t}=-\frac{1}{2} \frac{\partial^{2} \Psi}{\partial \phi^{2}}
$$




$$
\begin{gathered}
\dot{R}=-R^{2} \Pi \\
\dot{\Pi}=-\frac{R \Pi^{2}}{2}+\frac{3\left\langle\hat{p}^{2}\right\rangle}{2 R}+72 K R^{3}
\end{gathered}
$$

subject to the constraint

$$
-\frac{\Pi^{2}}{24 R}+\frac{\left\langle\hat{p}^{2}\right\rangle}{24 R^{3}}-6 K R=0
$$

where

$$
\left\langle\hat{p}^{2}\right\rangle=-\int_{-\infty}^{\infty} \Psi^{*} \frac{\partial^{2}}{\partial \phi^{2}} \Psi \mathrm{d} \phi
$$

and the phase condition $\alpha=-6 K R$ has been used for simplicity. These equations are similar in structure to those obtained by Kim in analyzing the Friedmann universe filled with a massless scalar field in the context of semiclassical gravity [33].

The wave equation (39) in fact decouples from the others and takes the same form as the 1dimensional Schrödinger equation for a free non-relativistic particle. Hence $\left\langle\hat{p}^{2}\right\rangle$ is preserved under evolution. It follows that the solution for $R$ can be generated from the classical solution (33) by replacing $|p|$ with $\sqrt{\left\langle\hat{p}^{2}\right\rangle}$. Like the classical case, there is no singularity avoidance and $R$ can become arbitrarily close to zero for all possibilities of $K$. Unlike the classical case, however, it is possible to envisage a wave packet with zero mean scalar field and momentum $(\langle\phi\rangle=\langle\hat{p}\rangle=0)$ but nonzero deviations $\left(\left\langle\phi^{2}\right\rangle>0\right.$ and $\left.\left\langle\hat{p}^{2}\right\rangle>0\right)$. In this case, the evolution of the Friedmann universe can be thought of as being 'purely quantum-driven'.

\section{Nonlinear quantum cosmology of the Bianchi type}

The foregoing Friedmann universe with a scalar field was quantized while maintaining the classical nature of the scale factor which carries the gravitational degree of freedom of the universe. In this section, two vacuum cosmological models, of Bianchi types I and IX, will be examined. Both belong to a wider class of homogeneous but anisotropic spacetimes [34. In Misner's seminal paper [35], the classical dynamics and quantization of Bianchi I and IX universes in the context of ADM quantization has been studied in detail. Below we will give derivations of the quantum evolution equations for these two cosmological models and comment on the consequences. The emphasis will be placed on the nonlinearity inherent in the derived quantum systems.

Just as in the Friedmann universe, the geometrical description of the Bianchi I and IX models involves the lapse function $N(t)$ and scale factor $R(t)$. Two additional functions $\beta_{+}(t)$ and $\beta_{-}(t)$ are used, to allow for the dynamical anisotropy of the spacial hypersurface at any coordinate time $t$. Following Misner's notation we consider a spacetime metric of the form

$$
\mathrm{d} s^{2}=-N^{2} \mathrm{~d} t^{2}+R^{2}\left(e^{2 \beta}\right)_{i j} \sigma_{i} \sigma_{j}
$$

for $i, j=1,2,3$, where $\sigma_{i}$ denote some basis 1 -forms of the spatial hypersurface and $\beta$ is a traceless matrix with elements given by

$$
\beta_{i j}=\operatorname{diag}\left(\beta_{+}+\sqrt{3} \beta_{-}, \beta_{+}-\sqrt{3} \beta_{-},-2 \beta_{+}\right) .
$$

The constant time spacial hypersurface for the Bianchi I universe is open and has an Abelian homogeneity group. Thus $\sigma_{i}$ are chosen so that $\mathrm{d} \sigma_{i}=0$ and can be simply expressed as $\sigma_{1}=\mathrm{d} x, \sigma_{2}=$ 
$\mathrm{d} y, \sigma_{3}=\mathrm{d} z$, for $-\infty<x, y, z<\infty$. The homogeneity group of the closed spacial hypersurface for the Bianchi IX universe is that of $\mathrm{S}^{3}$. Therefore the basis 1-forms $\sigma_{i}$ are chosen to satisfy the structure equations

$$
\mathrm{d} \sigma_{i}=\frac{1}{2} \epsilon_{i j k} \sigma_{j} \wedge \sigma_{k}
$$

and can be expressed explicitly as

$$
\sigma_{1}=\sin \psi \mathrm{d} \vartheta-\cos \psi \sin \vartheta \mathrm{d} \varphi, \sigma_{2}=\cos \psi \mathrm{d} \vartheta+\sin \psi \sin \vartheta \mathrm{d} \varphi, \sigma_{3}=-\mathrm{d} \psi-\cos \vartheta \mathrm{d} \varphi
$$

for $0 \leq \vartheta \leq \pi, 0 \leq \varphi<2 \pi$ and $0 \leq \psi<4 \pi$.

The classical dynamics of the models is generated by the Dirac-ADM action subject to the homogeneity condition for all metric functions. Up to an overall factor, the resulting Lagrangian takes the form

$$
L\left(\beta_{ \pm}, \dot{\beta}_{ \pm}, R, \dot{R}, N\right)=-\frac{6 R}{N} \dot{R}^{2}+\frac{6 R^{3}}{N}\left(\dot{\beta}_{+}^{2}+\dot{\beta}_{-}^{2}\right)+N R^{3} \mathcal{R}
$$

where $\mathcal{R}$ denotes the scalar curvature on the spatial hypersurface. It can be conveniently expressed as

$$
\mathcal{R}=\frac{3}{2 R^{2}}(1-V)
$$

where

$$
V=1
$$

for the Bianchi I model (such that $\mathcal{R}=0$ ) and

$$
\begin{aligned}
& V=V\left(\beta_{+}, \beta_{-}\right)= \\
& 1+\frac{1}{3} e^{-8 \beta_{+}}+\frac{1}{3} e^{4\left(\beta_{+}+\sqrt{3} \beta_{-}\right)}+\frac{1}{3} e^{4\left(\beta_{+}-\sqrt{3} \beta_{-}\right)}-\frac{2}{3} e^{4 \beta_{+}}-\frac{2}{3} e^{-2\left(\beta_{+}+\sqrt{3} \beta_{-}\right)}-\frac{2}{3} e^{-2\left(\beta_{+}-\sqrt{3} \beta_{-}\right)}
\end{aligned}
$$

for the Bianchi IX model. By introducing the conjugate momenta

$$
\begin{gathered}
\Pi:=\frac{\partial L}{\partial \dot{R}}=\frac{-12 R}{N} \dot{R} \\
p_{ \pm}:=\frac{\partial L}{\partial \dot{\beta}_{ \pm}}=\frac{12 R^{3}}{N} \dot{\beta}_{ \pm}
\end{gathered}
$$

we can construct the Hamiltonian $H\left(\beta_{ \pm}, p_{ \pm}, R, \Pi, N\right):=\Pi \dot{R}+p_{+} \dot{\beta}_{+}+p_{-} \dot{\beta}_{-}-L=N \mathcal{H}$ where

$$
\mathcal{H}\left(\beta_{ \pm}, p_{ \pm}, R, \Pi\right)=-\frac{\Pi^{2}}{24 R}+\frac{1}{24 R^{3}}\left(p_{+}^{2}+p_{-}^{2}\right)+\frac{3 R}{2}(V-1) .
$$

From (54) the canonical equations of motion can be derived and take a simpler form using the choice $N=12 R^{3}$ as in the Friedmann case. In this gauge, denoting $\partial_{ \pm}:=\partial_{\beta_{ \pm}}$, one obtains:

$$
\begin{gathered}
\dot{\beta}_{ \pm}=p_{ \pm} \\
\dot{p}_{ \pm}=-18 R^{4} \partial_{ \pm} V \\
\dot{R}=-\Pi R^{2}
\end{gathered}
$$




$$
\dot{\Pi}=\frac{\Pi^{2} R}{2}+\frac{3}{2 R}\left(p_{+}^{2}+p_{-}^{2}\right)-18 R^{3}(V-1)
$$

and the Hamiltonian constraint

$$
-\frac{1}{2} \Pi^{2} R^{2}+\frac{1}{2}\left(p_{+}^{2}+p_{-}^{2}\right)+18 R^{4}(V-1)=0 .
$$

The analogy between the Bianchi and Friedmann models should be apparent. Specifically by comparing the Lagrangian in (48) with that in (23) we see that the kinetic terms in both cases share the same structure with the functions $\beta_{ \pm}$resembling the scalar field $\phi$.

For the Bianchi I model with $V=1$, both $p_{+}$and $p_{-}$are constants of motion. By introducing

$$
p:=\sqrt{p_{+}^{2}+p_{-}^{2}}
$$

the solution for $R$ may be generated from the scale factor in the Friedmann model with zero curvature, i.e. $K=0$, given in (33). This yields

$$
R^{2}=\frac{p}{6} e^{2 p t}
$$

for $-\infty<t<\infty$, from which $\Pi$ can be evaluated using (57). The scale factor (61) together with $N=12 R^{3}$ and arbitrary constants $p_{ \pm}$is equivalent to the Kasner solution for the Bianchi I universe 36 .

The quantization of the Bianchi models can be performed once the dynamical variables to be quantized are decided. Guided by the analogous roles played by $\beta_{ \pm}$and $\phi$, we choose to turn $\beta_{ \pm}$ into quantum variables and introduce their wavefunctions $\Psi\left(\beta_{ \pm}, t\right)$. The inner product of two such wavefunctions $\Psi_{1}, \Psi_{2}$ can be defined to be

$$
\left\langle\Psi_{1}, \Psi_{2}\right\rangle:=\int_{-\infty}^{\infty} \int_{-\infty}^{\infty} \Psi_{1}^{*} \Psi_{2} \mathrm{~d} \beta_{+} \mathrm{d} \beta_{-} .
$$

We are concerned only with normalized wavefunctions $\Psi$ satisfying $\langle\Psi, \Psi\rangle=1$ so that $\langle\hat{o}\rangle=\langle\Psi, \hat{o} \Psi\rangle$ for any operator $\hat{o}$.

As before, the scale factor $R$ will act as the classical time that evolves with respect to the coordinate time $t$. The coupling between these quantum and classical variables follow from (18)-(22) applied in the current setting. In accordance with procedures leading to (39)-(42), we perform the 'kinematicdynamic' split

$$
\mathcal{H}=\mathcal{H}^{\mathrm{K}}+\mathcal{H}^{\mathrm{D}}
$$

where

$$
\begin{gathered}
\mathcal{H}^{\mathrm{K}}=-\frac{\Pi^{2}}{24 R} \\
\mathcal{H}^{\mathrm{D}}=\frac{1}{24 R^{3}}\left(p_{+}^{2}+p_{-}^{2}\right)+\frac{3 R}{2}(V-1)
\end{gathered}
$$

and construct the operator

$$
\hat{h}=-\frac{1}{2}\left(\partial_{+}^{2}+\partial_{-}^{2}\right) \Psi+18 R^{4}(V-1)
$$

from $\mathcal{H}^{\mathrm{D}}$ with the substitutions $p_{ \pm} \rightarrow \hat{p}_{ \pm}:=-\mathrm{i} \partial_{ \pm}$.

By using the conditions $N=12 R^{3}$ and $\alpha=0$ we arrive at the following nonlinear evolution equations

$$
\mathrm{i} \partial_{t} \Psi=-\frac{1}{2}\left(\partial_{+}^{2}+\partial_{-}^{2}\right) \Psi+18 R^{4}(V-1) \Psi
$$




$$
\begin{gathered}
\dot{R}=-\Pi R^{2} \\
\dot{\Pi}=\frac{\Pi^{2} R}{2}+\frac{3}{2 R}\left\langle\hat{p}_{+}^{2}+\hat{p}_{-}^{2}\right\rangle-18 R^{3}(\langle V\rangle-1)
\end{gathered}
$$

subject to the constraint

$$
-\frac{1}{2} \Pi^{2} R^{2}+\frac{1}{2}\left\langle\hat{p}_{+}^{2}+\hat{p}_{-}^{2}\right\rangle+18 R^{4}(\langle V\rangle-1)=0
$$

for $\Psi\left(\beta_{ \pm}, t\right), R(t)$ and $\Pi(t)$.

For the quantized Bianchi I model $(V=1)$, the decoupled wave equation (67) has the same structure as the Schrödinger equation for a free non-relativistic particle in 2-dimensions as expected. Hence $\left\langle\hat{p}_{+}^{2}+\hat{p}_{-}^{2}\right\rangle$ is preserved under evolution and the solution for $R$ can be generated from the

classical solution (61) by replacing $p$ with $\sqrt{\left\langle\hat{p}_{+}^{2}+\hat{p}_{-}^{2}\right\rangle}$. Clearly as $t \rightarrow-\infty$ the scale factor tends to zero, i.e. $R \rightarrow 0$, where the spacetime becomes singular. Using the above properties, one may demonstrate explicitly how this universe may be driven by quantized geometry described by a wave packet with zero means of the dynamical gravitational fields and their momenta $\left(\left\langle\beta_{ \pm}\right\rangle=\left\langle\hat{p}_{ \pm}\right\rangle=0\right)$ but with nonzero deviations $\left(\left\langle\beta_{+}^{2}+\beta_{-}^{2}\right\rangle>0\right.$ and $\left.\left\langle\hat{p}_{+}^{2}+\hat{p}_{-}^{2}\right\rangle>0\right)$.

For the quantized Bianchi IX model where $V$ is given in (51), equations (67)-(70) constitute a system of fully coupled nonlinear integro-partial differential equations. In the absence of explicit solutions it is not possible to analyze the dynamical behaviour of this model in detail. Nonetheless, it is worth noting two generic properties that one may conclude from this system. First, it can be shown that $V$ is nonnegative with its minimum $V=0$ taking place at $\beta_{ \pm}=0$. (In fact $V \approx 8 \beta_{+}^{2}+8 \beta_{-}^{2}$ for $\left|\beta_{ \pm}\right| \ll 1$. For further details of $V$, see [35].) It follows that the term $V-1$ in $\mathcal{H}$ can become negative which could be problematic for ADM quantization due to a square root taking procedure. The proposed quantization scheme is free from this problem, as exemplified in (67)- (70) where no square roots are involved. Secondly, although the scale factor is treated as a classical variable it is not used as an evolution parameter. As such, the quantum evolution will not come to a halt at the 'maximum hypersurface' where $\dot{R}=0$ classically. By comparison, the pathology associated with time integration across such a hypersurface could be suffered by both ADM quantization and Dirac quantization using Kuchař's embedding variables [11].

\section{Conclusions and discussions}

By virtue of its action principle, we have reformulated the Schrödinger equation in non-relativistic quantum mechanics into a parametric form. In this formalism the Newtonian time acts as a kinematic variable that evolves with respect to a general time coordinate while coupling to a quantum wavefunction in a semiclassical fashion. A gratifying feature of this description is that the parametrized action decomposes into a kinematic part and dynamic part. The former part of the action depends only on classical variables and takes a canonical form. Therefore, by preserving this form, one may perform a canonical transformation that redefines the kinematic time and its conjugate momentum. This suggests a natural extension of the proposed quantization method by accommodating a general expression of the kinematic time and its conjugate momentum that enter into the kinematic action. A quantum description obtained from this generalization is no longer reducible to the standard Schrödinger equation. Instead, it leads to a theory for nonlinear quantum evolution previously proposed based on 
heuristic arguments [28. In spite of the breakdown of the principle of superposition in time evolution, the resulting theory belongs to a class of nonlinear quantum theories admitting both probabilistic interpretation and a Hilbert space with Euclidean norm 37, 38.

These properties have indeed been demonstrated in the quantization of three types of cosmological models in this paper. The first model we considered was the Friedmann universe with a massless scalar field. The model consists of both gravitational and matter degrees of freedom, carried by the scale factor and scalar field respectively. Although classically these two variables take the same status as dynamical variables, given the physical origin of this model, we believe that it is matter that should be quantized thereby making the scale factor the intrinsic geometric time. In doing so we have not treated the scale factor merely as an evolution parameter as in certain alternative quantization methods. One obvious benefit of allowing the intrinsic geometric time to evolve as a classical variable is so that the theoretical description is not confined to scenarios with monotonically ascending geometric time. This avoids the diverse choice of time variables for different curvature parameters if the same cosmological model was quantized using the ADM method [32. It might be perceived at this point that our approach only applies to the quantization of matter in classical spacetime. To explicitly demonstrate it is not the case, we then considered the Bianchi I and IX cosmological models describing the evolution of an 'empty' universe. No matter fields are present there and one must decide which gravitational degrees of freedom should be quantized. In section 4 the two metric functions representing the anisotropy of the universe have been chosen for this purpose. Arguably this is the most appropriate choice due to the physical interpretation of these two functions as the gravitational 'waves' in the cosmological model. These considerations suggest that in extending the proposed quantization to quantum gravity, a primary concern is to determine which two gravitational field components are to be quantized so that the remaining four fields will act as classical kinematic variables. Unlike Kuchař's embedding variables, however, our approach does not require that the momenta of the kinematic variables to be resolved in the Hamiltonian and momentum constraints. In contrast, it may be speculated that if gravity were to be quantized based on the proposed quantization then the corresponding action integral should contain a kinematic part of the form

$$
S^{\mathrm{K}}\left[X^{A}, P_{A}, N^{A}\right]=\int \mathrm{d} t \int \mathrm{d}^{3} x\left\{P_{A} \dot{X}^{A}-N^{A} \mathcal{H}_{A}^{\mathrm{K}}\left(X^{B}, P_{B}\right)\right\}
$$

with $A, B=0,1,2,3$ where $N^{A}$ are Lagrangian multipliers proportional to the lapse and shift functions, $X^{A}$ and $P_{A}$ denote the gravitational fields and their momenta to act as classical kinematic variables and $\mathcal{H}_{A}^{\mathrm{K}}\left(X^{B}, P_{B}\right)$ are some expressions, not necessarily linear in $P_{A}$, to be determined. If such a split can be found uniquely in the full theory, then the classification of the geometrodynamical variables into kinematic and dynamic sets will follow naturally. In contrast, the classifications of variables in the truncated cosmological models in sections 3 and 4 have been partly guided by physical intuition.

A main thrust for this work has been the preservation of unitarity in quantizing physical systems with generic nonlinear kinematical structure. Of course, there exist other model-dependent strategies for unitary evolution within the linear quantum formulation, e.g. [21]. In a broader context, even the principle of unitarity can be debated by relaxing the probabilistic interpretation. Some have suggested that it is at best an approximate concept [33, 40. However, a more conclusive argument on the probabilistic interpretation of quantum gravity does not appear to be available at present. The non-unitary Wheeler-DeWitt equation is thought to be capable of describing a tunnelling [41. or topology-changing [42] wavefunction in certain models of the early universe. To what extent these theoretical scenarios can be accommodated or precluded by our approach would form a subject for future investigation. 
Although the proposed framework is somewhat reminiscent of semiclassical gravity original developed by Møller and Rosenfeld [39] as an exact theory, where all gravitational degrees of freedom are classical, it is worth stressing that we seek to quantize two gravitational field components. On the other hand, the remaining kinematic variables in our approach are classical by construction and therefore do not arise from a decoherence process required by Kiefer's approach to semiclassical gravity as an approximation to quantum gravity [40].

Before further potential implications of this work on quantum gravity can be contemplated seriously, several pressing issues ought to be addressed first, regarding the extension of the current quantum mechanical approach to a field-theoretical description. In this respect, it would be profitable to investigate the quantum dynamics of systems such as scalar fields in curved spacetime and gravitational wave spacetimes [43]. Progress made along these lines will pave the way for the analysis of a number of midisuperspace models where Dirac quantization using Kuchař's method has not been successfully applied. Results from research described above will be reported elsewhere.

\section{Acknowledgments}

We are most grateful to A Balanov, D A Burton, C J Isham, N Janson, J Louko, R Maartens, M A $\mathrm{H}$ MacCallum and R W Tucker for fruitful conversations and helpful comments on aspects in general relativity, quantum gravity, cosmology and nonlinear dynamical systems. Thanks are also due to the referees who drew our attention to works in [20, 21, 27, 33, 40, 41, 42. The research has been carried out under partial financial support from the EPSRC.

\section{References}

[1] Dirac P A M 1958 Proc. Roy. Soc., London Ser. A 246 326; 333; 1959 Phys. Rev. 114924

[2] Arnowitt R, Deser S and Misner C W 1962 Gravitation: An Introduction to Current Research ed L Witten (New York: Wiley)

[3] Wheeler J A 1962 Geometrodynamics (New York: Academic Press); 1968 Battelle Rencontres ed C M DeWitt and J A Wheeler (New York: Benjamin)

[4] DeWitt B S 1967 Phys. Rev. 1601113

[5] Teitelboim C 1973 Ann. Phys. NY 79 542; Hojman S A, Kuchař K and Teitelboim C 1973 Nature Phys. Sci. 245 97; Hanson A J, Regge T and Teitelboim C 1974 Constrained Hamiltonian Systems (Princeton: Princeton University Press)

[6] Baierlein R F, Sharp D H and Wheeler J A 1962 Phys. Rev. 1261864

[7] Kuchař K 1971 Phys. Rev. D 4955

[8] Kuchař K V 1972 J. Math. Phys. 13768

[9] Kuchař K 1976 J. Math. Phys. 17 777; 792; 801

[10] Kuchař K V 1981 Quantum Gravity 2: Second Oxford Symposium ed C J Isham, R Penrose and D W Sciama (Oxford: Clarendon)

[11] Kuchař K V 1992 Proc. 4th Canadian Conf. on General Relativity and Relativistic Astrophysics ed G Kunstatter, D E Vicent and J G Williams (Singapore: World Scientific)

[12] Isham C J and Kuchař K V 1985 Ann. Phys. NY 164 288; 316 
[13] Kuchař K V 1994 Phys. Rev. D 503961

[14] Berger B K, Chitre D M, Moncrief V E and Nutku Y 1972 Phys. Rev. D 52467

[15] Lund F 1973 Phys. Rev. D 83247

[16] Unruh W G 1976 Phys. Rev. D 14870

[17] Romano J D 1997 Phys. Rev. D 55 1112; 1995 gr-qc/9501015

[18] Hájiček P 1986 Phys. Rev. D 341040

[19] Hájiček P 1999 Talk at the 19th Texas Symposium of Relativistic Astrophysics and Cosmology, Paris, 1998 gr-qc/9903089.

[20] Hájíček P and Kijowski J 2000 Phys. Rev. D 61 024037; 62044025

[21] Hájiček P 2000 Nucl. Phys. B (Proc. Suppl.) 88 114; Hájiček P and Kiefer C 2001 Nucl. Phys. B 603 531; Hájiček P 2000 Nucl. Phys. B 603 555; Hájiček P and Kiefer C 2001 Int. J. Mod. Phys. D 10775

[22] Louko J, Whiting B F and Friedman J L 1998 Phys. Rev. D 572279

[23] Torre C G 1992 Phys. Rev. D 46 R3231

[24] Torre C G and Varadarajan M 1999 Class. Quantum Grav. 162651

[25] Schön M and Hájíček P 1990 Class. Quantum Grav. 7861

[26] Isham C J 1993 Canonical Gravity: From Classical to Quantum ed J Ehlers and H Friedrich (Berlin: Sringer-Verlog)

[27] Barvinsky A O and Krykhtin V 1993 Class. Quantum Grav. 10, 1957; Barvinsky A O Class. Quantum Grav. 101985

[28] Wang C 2003 Class. Quantum Grav. 203151

[29] DeWitt B S 1964 Relativity, Groups and Topology ed C DeWitt and B DeWitt (Blackie and Son, London)

[30] Kibble T W B and Randjbar-Daemi S 1980 J. Phys. A 13141

[31] Kibble T W B 1978 Commun. Math. Phys. 64 73; 1979 Commun. Math. Phys. 65 189; 1981 Quantum Gravity 2: Second Oxford Symposium ed C J Isham, R Penrose and D W Sciama (Oxford: Clarendon)

[32] Blyth W F and Isham C J 1975 Phys. Rev. D 11768

[33] Kim S P 1995 Phys. Rev. D 52 3382; 1997 Phys. Rev. D 557511

[34] Ryan M P and Shepley L C 1975 Homogeneous Relativistic Cosmologies (Princeton: Princeton University Press)

[35] Misner C W 1969 Phys. Rev. 1861319

[36] Kasner E 1921 Am. J. Math. 43217

[37] Mielnik B 1974 Commun. Math. Phys. 37221

[38] Weinberg S 1989 Ann. Phys. NY 194336

[39] Møller C 1962 Les Theories Relativistes de la Gravitation (Paris: CNRS); Rosenfeld L 1963 Nucl. Phys. 40353 
[40] Kiefer C 1992 Phys. Rev. D 46 1658; 47 5414; 1994 in Canonical gravity-from classical to quantum ed J Ehlers and H Friedrich (Berlin: Springer) (Preprint gr-qc/9312015)

[41] Levkov D, Rebbi C and Rubakov V A 2002 Phys. Rev. D 66 083516; Hong J, Vilenkin A and Winitzki S 2003 Phys. Rev. D 68 023520; 023521

[42] Kim S P 1999 Class. Quantum Grav. 163987

[43] Wang C H-T and Wang R C-H In preparation 